\title{
To treat or not to treat? and How to treat? Two questions whose answers are far removed from evidence informed practice in child psychiatry
}

\author{
Celso Arango
}

Published online: 7 August 2013

(C) Springer-Verlag Berlin Heidelberg 2013

Decision making in medicine is not subject to mathematical or physical rules and goes well beyond the existence of evidence. In this issue, based on previous research on the variables that influence clinicians' decisions about ADHD medication, Marina Danckaerts et al. discuss the reliability of the Influences on Prescribing for ADHD Questionnaire (IPAQ) and explore the relationship between questionnaire scores and clinician background and characteristics (Danckaerts et al., this issue). Sixty-eight clinicians from Belgium and the UK were interviewed. Despite the small sample size the authors identified four groups of prescribers-pro-psychosocial; medication focused; unsystematic; and response optimizers-with important differences between pediatricians and psychiatrists. Regardless of their specialty participants received high overall ratings on the rule-based approach subscale, suggesting that clinicians in this sample believe that their prescribing decisions are currently informed by best practice and published guidelines. Unfortunately, I do not believe this is the rule for many treatment decisions in the mental health field.

It is my belief that there is nothing more harmful to our medical specialty than the lack of evidence informed practice. It is clear that prescription rates vary widely by country, region and even within the same city depending on the clinical staff in the absence of differences in prevalence rates for the mental disorders treated. In my country it is sometimes the case that prescription rates and the use of specific therapeutic interventions actually vary with the

\section{Arango ( $\square)$}

Child and Adolescent Psychiatry Department, CIBERSAM, Instituto de Investigación Sanitaria Gregorio Marañón, IiSGM, School of Medicine, Hospital General Universitario Gregorio Marañón, Universidad Complutense, Madrid, Spain e-mail: carango@hggm.es political views of the psychiatrist (left-leaning voters use less pharmacotherapy and want to prohibit ECT while right-leaning voters are less inclined to use dynamic psychotherapy) (sic). Why do national health services provide treatments for which there is no evidence solely on the basis that professionals prefer them to other available treatments? This is something that public medicine should not allow and cannot afford. We should follow the example of the UK's NICE with regard to evidence-based recommendations on public health system treatment coverage.

Some mental health clinicians dislike the idea that only evidence informed practice treatments would be offered by national health services, deeming this a simplistic proposal that would make the field more rigid and unable to allow for personal preferences. Of course this does not mean that we should sacrifice one of our best added values compared with the majority of other medical disciplines: the importance of the human relationships and psychosocial aspects in dealing of the suffering of our patients. It is the mixture of a warm and sensitive approach to our patients and our scientific background that should guide our interventions and recommendations for achieving clinical excellence. And this is even more important when dealing with individuals who are more amenable to change and more subject to improvement through different treatment approaches as is the case with the pediatric population [1,2]. As Letamendi said long ago, it is not about disorders but about the people suffering from those disorders. However, those who apply "their preferred treatment" (e.g., dynamic therapy, behavioral therapy, pharmacotherapy) to all patients are very far removed from this principle.

The practice of medicine is about taking decisions under conditions of uncertainty which means taking risks. As medicine is not an exact science, risk taking is inherent to our profession. In fact it has been claimed that most 
published research findings are false and that research findings may often simply be accurate measures of the prevailing bias [4]. Therefore, there is no completely safe environment in which we can be sure of taking the right decision for a given patient. Nor can we claim that making the right decisions in mental health is mostly a matter of common sense (like the common sense view that increasing security in airports is good for everyone when it has been shown that the resulting increase in road fatalities has caused more deaths that all terrorist attacks in the history of aviation). However, an intervention supported by evidence (howsoever biassed) would always be better than one based on gut feelings or because "this is what I have always believed was the way to do things".

To preclude patients from receiving an efficacious treatment based on preconceptions and "philosophical" interests is unethical and should not be permitted by the health authorities in each country. That clinicians would use one therapeutic intervention or another (psychotherapeutic or pharmacological) with the different patients they see regardless of the evidence smacks more of religion than science. We have recently seen movements such as the denial of autism as a brain disorder in France that are truly harmful and result in some patients not receiving treatments that clearly improve outcome and quality of life for them and their families [3]. It is not about following guidelines (some of which have very little supporting evidence) but about not always trying to be different from the rest of medicine... in the wrong way.

\section{References}

1. Arango C (2012) Someone is not listening to the facts: there is little psychiatry outside child and adolescent psychiatry. Eur Child Adolesc Psychiatry 21(9):475-476

2. Parellada M (2013) Why psychogeriatrics starts right after adolescence. Eur Child Adolesc Psychiatry 22(7):391-393

3. Parellada M, Penzol MJ, Pina L, Moreno C, González-Vioque E, Llorente C, Zalsman G, Arango C. The neurobiology of autism spectrum disorders. Eur Psych (in press)

4. Ioannidis JP (2005) Why most published research findings are false. PLoS Med 2(8):e124 